\title{
Factors associated with immunotherapy selection in patients with advanced melanoma
}

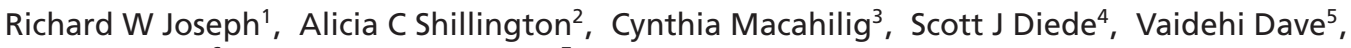 \\ Qing Harshaw6 \& Frank Xiaoqing Liu*,7 \\ ${ }^{1}$ Division of Hematology/Oncology, Department of Internal Medicine, Mayo Clinic, Jacksonville, FL, USA \\ ${ }^{2}$ Health Economics and Outcomes Research, EPI-Q Inc. Oakbrook, IL, USA \\ ${ }^{3}$ Medical Data Analytics, Parsippany, NJ, USA \\ ${ }^{4}$ Oncology Clinical Research, Merck \& Co., Inc., Kenilworth, NJ, USA \\ ${ }^{5}$ Medical Data Analytics, Parsippany, NJ, USA \\ ${ }^{6}$ Statistics and Analytics, EPI-Q Inc. Oakbrook, IL, USA \\ ${ }^{7}$ Center for Observational and Real World Evidence, Merck \& Co., Inc., Kenilworth, NJ, USA \\ *Author for correspondence: Tel.: +1 267305 1265; Xiaoging.liu@merck.com
}

Aim: To explore factors associated with pembrolizumab (PEMBRO) versus ipilimumab + nivolumab (IPI+NIVO) selection in advanced melanoma. Materials \& methods: Total of 12 academic and satellite clinics contributed to this study. Descriptive and logistic regression analyses were conducted to explore associations between clinical characteristics and treatment choice. Results: Total of 400 patients were included: 200 PEMBRO and 200 IPI+NIVO. Patients were significantly more likely to receive PEMBRO versus IPI+NIVO if they had poorer Eastern Cooperative Oncology Group score, 2-4 versus 0-1 (odds ratio [OR]: $6.6 ; 95 \% \mathrm{Cl}: 3.0-14.7)$, if they were PD-L1 positive (OR: $4.5 ; 95 \% \mathrm{Cl}: 1.9-10.4)$ or had BRAF wild-type tumor (OR: 2.2; $95 \% \mathrm{Cl}: 1.4-3.6$ ). Conclusion: Patient factors are significantly associated with treatment selection in advanced melanoma. Outcomes comparisons should take this into consideration.

First draft submitted: 27 September 2018; Accepted for publication: 23 October 2018; Published online: 23 November 2018

Keywords: advanced melanoma • immunotherapy • ipilimumab • nivolumab • pembrolizumab • real-world study - treatment selection

Melanoma afflicts 5.3\% of the people diagnosed with skin cancer in the USA [1]. In 2018, it is estimated that there will be 91,270 new cases of melanoma of the skin and an estimated 9320 people will die of this disease [2]. Metastatic melanoma in particular has historically been associated with a very poor prognosis. A 5-year survival rates in stage IV disease is no more than 20\% [1]. However, in recent years, innovative therapeutic strategies have demonstrated improved survival through the use of immuno or targeted therapies. PD-1 inhibitors are promising therapies for the treatment of unresectable or metastatic melanoma with superior overall survival (OS) [3-5]. Both pembrolizumab (PEMBRO; Merck \& Co., Inc., NJ, USA) and the combination of ipilimumab with nivolumab (IPI+NIVO; Bristol-Myers Squibb, NY, USA) are approved by the US FDA for the treatment of advanced melanoma [6,7]. When comparing PEMBRO with IPI in controlled trials, PEMBRO demonstrated prolonged progression-free survival (PFS) and OS with fewer high-grade adverse events (AEs) [3]. The NIVO administered in combination with IPI has demonstrated improved OS, PFS and higher rates of objective response than IPI alone [4].

While PEMBRO and IPI+NIVO are efficacious, clinical trials have demonstrated different benefit-risk profiles associated with each with reported grade 3 or 4 treatment-related AEs of 17 and 59\%, respectively [3-6]. It is not clear that how these differences in toxicity may impact advanced melanoma treatment selection. Studies that have surveyed preferences suggest that patients receiving adjuvant melanoma treatment are willing to accept greater toxicity for modest additional survival benefit $[8,9]$. A study of patient preferences in advanced melanoma suggests that while patients place high value on therapeutic outcomes, this is balanced with the risk of toxicity. In this study, optimizing OS was ranked as the most important treatment attribute in surveyed patients, followed closely by minimizing grade 3-4 toxicities/AEs. Clinicians when surveyed, far outranked the risk of AEs as important

Future 8 Medicine 
in treatment choice relative to OS [10]. However, no published evidence exists to understand if and how differing benefit-risk profiles impact real-world treatment selection in clinics that utilize both treatment strategies.

Therefore, the objective of this study was to conduct a retrospective chart review and analysis of patients with advanced melanoma to understand if patient demographic and disease characteristics can be identified that predict the treatment choice of PEMBRO versus IPI+NIVO.

\section{Patients \& methods}

\section{Data source}

This is an analysis of baseline data from an ongoing 3-year cohort study of randomly selected advanced melanoma treatment in patients treated in US-based academic and satellite centers. Total of 12 geographically dispersed centers (three from Northeast, four from Midwest, two from South and three from West) were identified and recruited from a national database of oncology providers in the USA. Physicians were recruited by telephone and underwent screening to confirm eligibility prior to acceptance as study investigators. To contribute patients, academic centers needed to treat a minimum of 15 advanced melanoma patients each year, and satellite centers needed to treat a minimum of seven. Sites must have reported both PEMBRO and IPI+NIVO available for use in any line of therapy (LOT) and agree to all study procedures. The participating physicians were enrolled into the study and signed the site agreement acknowledging and agreeing to study obligations. Sites were trained on standardized data collection, data dictionary and random sampling procedure. This study was sponsored by Merck \& Co. Sites were blinded to sponsor, and sponsor was blinded to sites.

\section{Study sample}

Patients were randomly sampled into two cohorts of 200 patients each. Cohorts consisted of patients receiving either PEMBRO or IPI+NIVO in any LOT administered for their advanced melanoma. Included in the cohorts were patients 18 years or older with histologically confirmed diagnosis of unresectable stage III or metastatic/stage IV melanoma (excluding uveal or ocular melanoma), who have received PEMBRO or IPI+NIVO in any LOT. Treatment must have been initiated between 1 January 2016 and 30 December 2017. Patients receiving investigational agents in any LOT and patients with incomplete medical records were excluded.

\section{Data collection}

Collected data at entry into the cohort study included demographics (age, gender, race, ethnicity, marital status, insurance coverage, highest education level, annual family income and employment status). Clinical characteristics at time of immunotherapy initiation included LOT, American Joint Committee on Cancer (AJCC) stage, M stage, presence of brain metastases, lactate dehydrogenase (LDH), Eastern Cooperative Oncology Group (ECOG) performance status, comorbidities including Charlson Comorbidity Index (CCI) and BMI and BRAF mutation testing prevalence and results, in addition to PD-L1 expression testing prevalence and results were captured. PD-L1 expression was recorded as positive if $>0 \%$.

\section{Standard protocol approvals, registrations \& patient consent}

Collected data were deidentified in compliance with the Health Insurance Portability and Accountability Act of 1996. Synthetic identifiers were created by investigators maintaining a patient-level crosswalk to protect the confidentiality of records. A central institutional review board (IRB) and compliance/privacy approval was obtained from New England IRB on 3 February 2017, prior to initiation of the study. As this was a study of existing deidentified data, waiver of informed consent was received.

\section{Statistical analysis}

Patient, clinical and treatment characteristics were summarized descriptively within each cohort using means (standard deviations [SD]), medians (ranges) for continuous data and proportions for categorical data. As this study was not randomized, characteristics were compared between cohorts in univariate analyses using student t-test or $\chi^{2}$ statistics to attempt to identify factors that may have led to selecting PEMBRO or IPI+NIVO focusing on those that might be associated with more severe illness including presence of metastasis, LDH elevation ECOG performance status, presence of mutation and PD-L1 expression and demographic. Characteristics differing between cohorts were discussed by investigators for theoretic plausibility related to PEMBRO versus IPI+NIVO selection and then models were created. 
A mixed effects logistic regression model was constructed to examine predictors of PEMBRO versus IPI+NIVO selection (binomial distribution). Mixed effects modeling (PROC GLIMMIX; SAS v9.4, NC, USA) was implemented, recognizing that data is multilevel, in that patients are nested in sites with characteristics that could affect treatment selection in addition to patient-specific factors [11]. Traditional regression equations do not consider random effects of patients being nested within institutions, and that local practice patterns within institutions might exert an effect on treatment selection beyond individual patient characteristics. This clustering or nested effect has the potential for too liberally interpreting the effects of parameters. To avoid this ecological fallacy, [12] for example, making assertions regarding macrolevel variables about microlevel relations, multilevel mixed effects models were used. Site was treated as a random effect and clinical parameters were treated as fixed effects. Variables with theoretically plausible association with treatment selection identified in univariate analysis were entered the model and removed if $\mathrm{p}>0.1$. For all statistical tests, $\alpha=0.05$ in a two-tailed fashion was implemented.

\section{Results}

Total of 400 patient records were included, 200 each from patients receiving PEMBRO and IPI+NIVO. Use of PEMBRO or IPI+NIVO was well balanced between sites (Supplementary Table). Regardless of cohort, patients were primarily Caucasian (94.5\%) aged 62 years at treatment initiation and 50.5\% were male. Most patients $(67.5 \%)$ were married with at least some post-high school education $(81.8 \%)$. Less than half $(45.0 \%)$ were still employed and there were no differences in family income level between cohorts with $25 \%$ having incomes exceeding US\$100,000 per annum. However, sites were unable to document family income in 35.3\% (Table 1).

More than half of all patients (61.5\%) were commercially insured. Most had prescription drug coverage through a commercial insurer $(61.0 \%)$, or through a government payer (36.7\%) (Table 2). None of these factors differed significantly between cohorts.

Both PEMBRO and IPI+NIVO were primarily administered as first-line therapy (84 and $87.5 \%$, respectively; $\mathrm{p}=0.436$ ). With respect to clinical characteristics at therapy initiation, cohorts were similar in AJCC and M stages, with 83.5 and $79 \%$, respectively having metastatic disease at treatment initiation. Brain metastases at treatment initiation were evident in 11.2 and $14.0 \%$, respectively and there were no significant differences in proportion of patients with elevated LDH from one- to two-times the upper level of normal (30.1 vs $23.7 \%$, PEMBRO vs IPI+NIVO, respectively), or elevated $\mathrm{LDH}$ greater than two-times the upper limit of normal (17.5 vs $14.4 \%$, PEMBRO vs IPI+NIVO, respectively). Comorbidity index was also similar between cohorts (2.9 vs 2.8). There were however significant differences between cohorts related to ECOG performance status at therapy initiation with the PEMBRO cohort having poorer ECOG status at treatment start, 29.5\% ECOG 2-4 versus 12.0\% (p $<0.001$ ) (Table 3).

Mutation testing was common in both cohorts, but more so in PEMBRO compared with IPI+NIVO with greater than $98 \%$ of testing performed prior to initiation of immunotherapy. When $B R A F$ testing was done (94.5\% of patients), a greater proportion of patients displayed wild-type (WT) tumor in the PEMBRO cohort versus IPI+NIVO (65.2 vs 50.3\%; $\mathrm{p}=0.003)$. PD-L1 expression testing was used less often than BRAF testing 65.0 versus $78.5 \%$, respectively, and when patients were tested, tumor PD-L1 expression was more evident in the PEMBRO versus IPI+NIVO cohorts (76.9 vs $63.1 \%$, respectively; $\mathrm{p}=0.011$ ). Of those positive, expression $\geq 50 \%$ was reported in $41.8 \%$ of PEMBRO and $37.9 \%$ in IPI+NIVO, a nonsignificant difference (Table 4).

A mixed effects model to predict the selection of PEMBRO over IPI+NIVO included ECOG 0 or 1 versus 2-4, tumor demonstrating PD-L1 expression, and the presence of BRAF WT tumor. The odds of receiving PEMBRO as opposed to IPI+NIVO were 6.6-times greater (95\% CI: 3.0-14.7) in those with poorer performance status, 4.5-times greater (95\% CI: 1.9-10.4) in tumors with positive PD-L1 expression, 2.2-times more likely (95\% CI: 1.4-3.6) and in patients with BRAF WT (Figure 1).

\section{Discussion}

This is the first study we are aware of identifying factors associated with immunotherapy selection in advanced melanoma patients in the real-world among factors prespecified by the investigators, including presence of metastasis, LDH elevation ECOG performance status, presence of mutation and PD-L1 expression and demographics. We identified three characteristics associated with selecting PEMBRO versus IPI+NIVO - ECOG performance status, positive PD-L1 expression. In patients with $B R A F$ mutated tumor, PEMBRO was less likely selected. Both cohorts were otherwise comparable with no significant differences with respect to demographics including age, gender, race/ethnicity, insurance status, employment status, annual family income, education or marital status. Patients 
Research Article Joseph, Shillington, Macahilig et al.

Table 1. Patient demographics at treatment initiation.

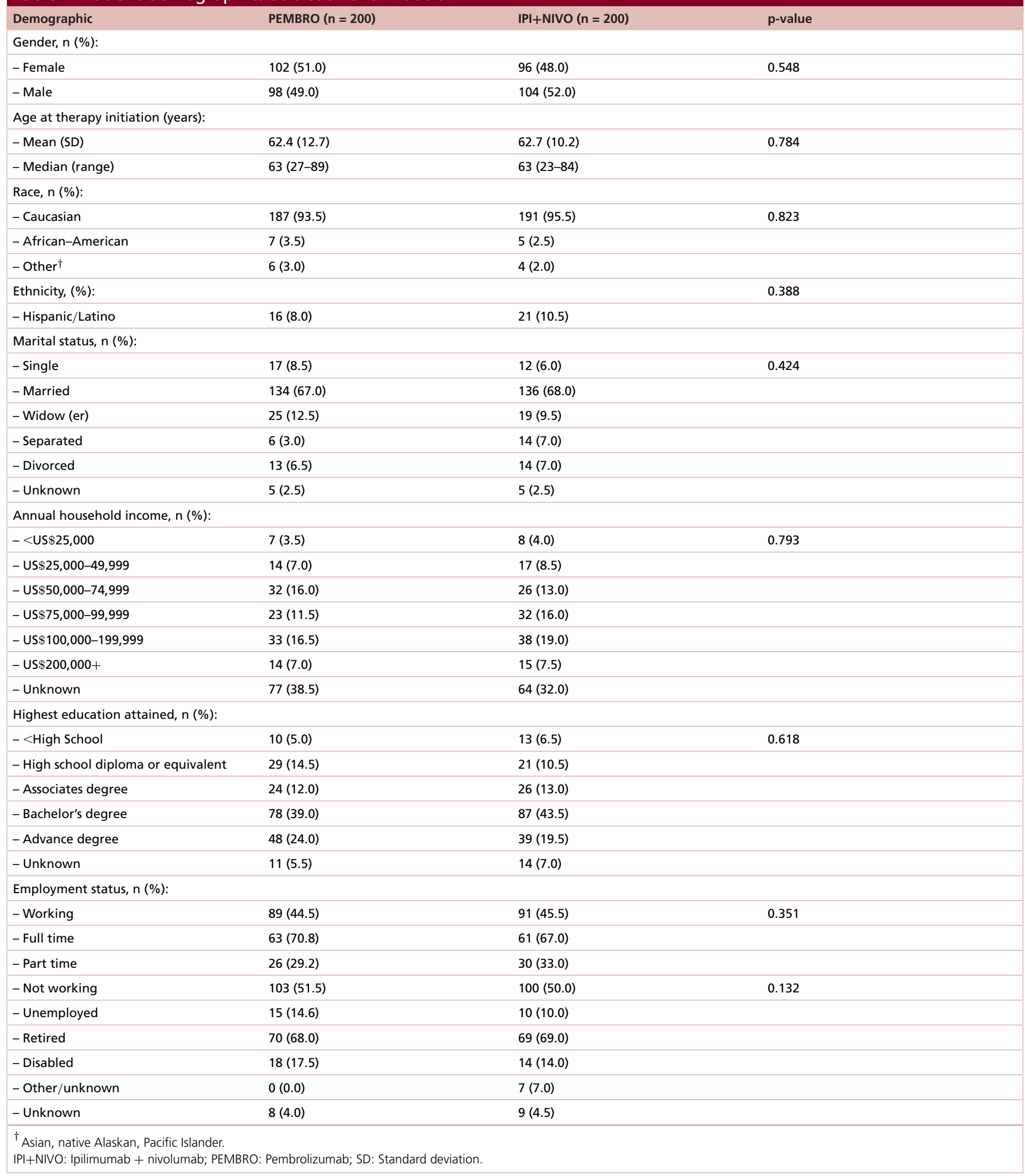


Table 2. Patient insurance coverage at treatment initiation.

\begin{tabular}{|c|c|c|c|}
\hline Insurance type & $\operatorname{PEMBRO}(n=200)$ & $I P I+N I V O(n=200)$ & p-value \\
\hline \multicolumn{4}{|c|}{ Current primary medical insurance coverage at therapy initiation, $n(\%)$ : } \\
\hline - HMO & $36(18.0)$ & $42(21.0)$ & 0.265 \\
\hline - PPO & $70(35.0)$ & $63(31.5)$ & \\
\hline Fee for service/indemnity: & $9(4.5)$ & $20(10.5)$ & \\
\hline - Medicare & $66(33.0)$ & $51(25.5)$ & \\
\hline - Medicaid & $15(7.5)$ & $18(9.0)$ & \\
\hline $\begin{array}{l}\text { Current secondary medical insurance } \\
\text { coverage at therapy initiation, } n(\%) \text { : }\end{array}$ & $\mathrm{n}=60$ & $\mathrm{n}=32$ & \\
\hline$-\mathrm{HMO}$ & $7(11.9)$ & $3(9.4)$ & 0.439 \\
\hline - PPO & $24(40.0)$ & $12(37.5)$ & \\
\hline Fee for service/indemnity: & $6(10.0)$ & $1(3.1)$ & \\
\hline - Medicare & $6(10.0)$ & $2(6.3)$ & \\
\hline \multicolumn{4}{|c|}{ Current primary drug coverage at therapy initiation, $\mathrm{n}(\%)$ : } \\
\hline$-\mathrm{HMO}$ & $35(17.5)$ & $41(20.5)$ & 0.421 \\
\hline - PPO & $75(37.5)$ & $64(32.0)$ & \\
\hline Fee for service/indemnity: & $9(4.5)$ & $20(10.0)$ & \\
\hline - Medicare & $63(31.5)$ & $52(26.0)$ & \\
\hline - Medicaid & $14(7.0)$ & $18(9.0)$ & \\
\hline - Other & $4(2.0)$ & $5(2.5)$ & \\
\hline $\begin{array}{l}\text { Current secondary drug coverage at } \\
\text { therapy initiation, } \mathrm{n}(\%) \text { : }\end{array}$ & $\mathrm{n}=57$ & $\mathrm{n}=30$ & \\
\hline - HMO & $6(10.5)$ & $3(10.0)$ & 0.412 \\
\hline
\end{tabular}

HMO: Health maintenance organization; IPI+NIVO: Ipilimumab + nivolumab; PEMBRO: Pembrolizumab; PPO: Preferred provider organization.

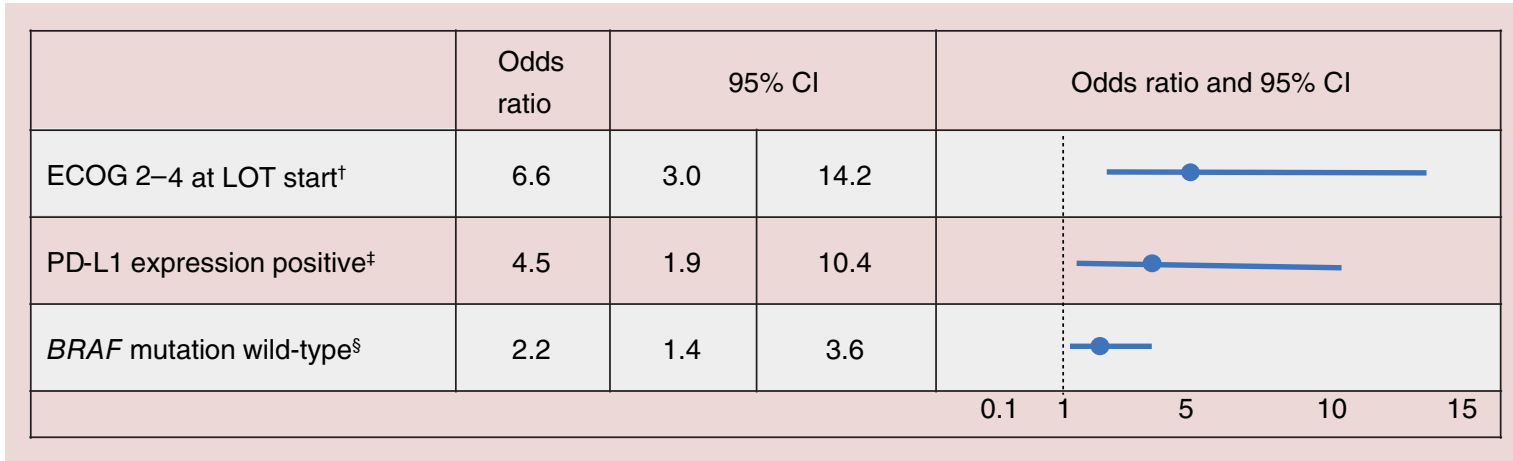

Figure 1. Factors predicting treatment with pembrolizumab versus ipilimumab plus nivolumab. Site treated as random effect.

$\dagger$ Reference condition ECOG $=0$ or 1 .

$¥$ Reference condition PD-L1 expression negative.

Reference condition BRAF positive.

ECOG: Eastern Cooperative Oncology Group; LOT: Line of therapy. 
Table 3. Clinical characteristics at treatment initiation.

\begin{tabular}{|c|c|c|c|}
\hline Characteristics & $\operatorname{PEMBRO}(n=200)$ & $I P I+N I V O(n=200)$ & p-value \\
\hline \multicolumn{4}{|l|}{ Line of therapy agent administered: } \\
\hline - First line & $168(84.0)$ & $175(87.5)$ & 0.436 \\
\hline - Second line & $31(15.5)$ & $23(11.5)$ & \\
\hline - Third line & $1(0.5)$ & $2(1.0)$ & \\
\hline \multicolumn{4}{|l|}{ AJCC stage at treatment initiation, $n(\%)$ : } \\
\hline$-111 \mathrm{~b}$ & $18(9.0)$ & $26(13.0)$ & 0.420 \\
\hline$-I V$ & $168(84.0)$ & $159(79.5)$ & \\
\hline \multicolumn{4}{|l|}{ M stage, n (\%): } \\
\hline$-m 1 a$ & $32(19.0)$ & $42(26.4)$ & 0.278 \\
\hline$-m 1 b$ & $29(17.3)$ & $24(15.1)$ & \\
\hline$-m 1 c$ & $107(63.7)$ & $93(58.5)$ & \\
\hline$-1-2$ ULN & $55(30.1)$ & $46(23.7)$ & \\
\hline - >two-times ULN & $32(17.5)$ & $28(14.4)$ & \\
\hline Charlson Comorbidity Index, mean (SD) & $2.9(2.0)$ & $2.8(1.6)$ & 0.619 \\
\hline \multicolumn{4}{|l|}{$\mathrm{ECOG}^{\dagger}, \mathrm{n}(\%)$} \\
\hline$-0-1$ & $141(73.4)$ & $176(88.0)$ & 0.001 \\
\hline$-2-4$ & $51(26.6)$ & $24(12.0)$ & \\
\hline \multicolumn{4}{|l|}{ BMI: } \\
\hline - Mean (SD) & $27.7(4.6)$ & $27.2(3.7)$ & 0.246 \\
\hline - Median (range) & $27.2(18.9-53.4)$ & $27.4(17.4-40.5)$ & \\
\hline
\end{tabular}

\begin{tabular}{|c|c|c|c|}
\hline Molecular characteristics & $\operatorname{PEMBRO}(n=200)$ & IPI+NIVO $(n=200)$ & $p$-value \\
\hline \multicolumn{4}{|l|}{ BRAF mutation, $\mathrm{n}(\%)$} \\
\hline - Negative & $118(59.0)$ & 99 (49.5) & \\
\hline - Not tested & $19(9.5)$ & $3(1.5)$ & \\
\hline PD-L1 expression, n (\%) & & & 0.000 \\
\hline - Negative & $30(15.0)$ & $58(29.0)$ & \\
\hline - Not tested & $70(35.0)$ & $43(21.5)$ & \\
\hline
\end{tabular}

were also similar relative to LOT in which PEMBRO or IPI+NIVO were administered, AJCC disease stage, M stage, prevalence of brain metastases and LDH elevation, each measured at the time of treatment initiation.

It might be anticipated that patients with a poor prognosis, including functional status impairments would be candidates for more aggressive therapy. However, our study found the opposite. Our study suggests that PEMBRO is used with greater frequency than IPI+NIVO in more frail patients, including those with an identified ECOG performance status of two or higher. Although ECOG status may not always be routinely documented in clinical practice outside the academic setting, our data suggests that in patients with noted limitations in performance, they are nearly six-times more likely to have received PEMBRO versus IPI+NIVO. Differences in benefit-risk profiles between PEMBRO and IPI+NIVO in advanced melanoma have been elucidated in trials, which may have 
impacted treatment selection in this patient group [3,4]. Higher rates of grade 3 or 4 treatment related AEs have been demonstrated with IPI+NIVO (59\%) than for PEMBRO (17\%) which may make PEMBRO a less toxic and preferable treatment for a more frail population. Our results are consistent with a recent discrete choice experiment in which clinicians were asked to rate attributes of therapy options for advance melanoma relative to treatment selection. In this study, oncologists ranked avoidance of grade 3-4 AEs as the most important factor in determining therapy selection, more important even than survival benefit [9].

Interestingly, nearly $72 \%$ of the patient sample in this study underwent PD-L1 expression testing. This is substantially higher than a recently published real-world study conducted in electronic medical record data in a community oncology setting. This study reported virtually no PD-L1 expression testing being utilized (two of 168 patients). The reason for this vast difference is not clear. Our sample being derived primarily from academic-based settings along with their satellite community centers may have led to higher rates of testing compared with a solely community setting. Also, the community-based study collected data on patients initiating treatment prior to 2016, whereas our current study collected data on patients initiating therapy from early 2016 through December 2017. Therefore, the results of this current study may reflect increased adoption of PD-L1 expression testing as the test is now available in standardized panels. When PD-L1 expression was identified, PEMBRO was selected four-times as often as IPI+NIVO. This finding is interpreted in the context of findings in the Checkmate 067 study which demonstrated that patients who are PD-L1 positive tend to benefit from single agent anti-PD-L1 therapy equally well as the combination of IPI and NIVO [4]. Therefore, physicians might prescribe the less toxic regimen when efficacy appears to be equivalent.

Our data demonstrates that PEMBRO was selected more than twice as often as IPI+NIVO in patients whose tumor was BRAF WT which was consistent with Cowley et al. [13]. It remains unclear why physicians favored PEMBRO over IPI+NIVO in BRAF WT patients; however, one possible explanation may be because in a subset and exploratory analysis of Checkmate 067 , IPI+NIVO appeared to have more activity in $B R A F$ mutant patients [4]. It should be noted that the frequency of $B R A F$ mutations reported here is in line with the $40-60 \%$ expected in a population of patients with primarily cutaneous melanomas [14].

\section{Limitations}

This study was conducted as a retrospective medical chart review, and therefore the results were limited by the completeness of the information that was recorded in those charts and data that was collected. Study parameters important in predicting therapy selection, such as ECOG performance status, are not always assessed in routine medical practice. However, as an assessment of frailty can be made using clinical judgement and patient objective presentation during a clinical consultation, this may or may not be a limitation in extrapolating our findings to routine practice. Although nearly $98 \%$ of all $B R A F$ mutation and PD-L1 expression testing was dated prior to therapy initiation, we have no way to confirm that results were viewed by clinicians prior to selecting PEMBRO versus IPI + NIVO. This study is limited by lack of data on tumor burden. Symptomatology from disease collected was limited to performance status. Additional symptoms and tumor burden may have impacted treatment selection. Also, melanoma subtypes (such as acral or uveal mucosal) are not represented and could be factors for choosing IPI+NIVO versus PEMBRO.

Although both PEMBRO and IPI+NIVO were approved by the US FDA prior to the start of the study period and all sites used both treatments, unobserved barriers to one treatment versus another (e.g., insurance prior authorizations, local practice patterns and site formularies) may have impacted treatment selection for periods of time during the study. Use of mixed effects modeling was selected to attempt to overcome this limitation. Also, only PEMBRO was studied as a single agent PD-1 inhibitor although nivolumab (Bristol-Myers Squibb) was also approved for monotherapy in this indication. As patient preference is not generally available during retrospective chart review but may have impacted treatment selection, we were unable to assess preference as a decision factor. This analysis did not include some clinical characteristics, like tumor disease bulk or aggressiveness that may have impacted treatment choice. Although this analysis did not specifically assess impact of treatment selection on real-world objective response, OS and PFS; however, the study is ongoing.

\section{Conclusion}

In this real-world study, there are differences in patients' characteristics between those prescribed PEMBRO or IPI+NIVO including patient performance status, $B R A F$ mutations and PD-L1 expression. Comparison of outcomes between treatments should take these differences into consideration. 
Summary points

- Pembrolizumab (PEMBRO) and ipilimumab plus nivolumab (IPI+NIVO) are approved in the USA for advanced melanoma.

- Randomized clinical trials have demonstrated different benefit-risk profiles associated with PEMBRO and IPI+NIVO that may impact treatment selection. However, how the treatments are selected in the real-world oncology clinical practices is unknown.

- Total of 400 adult advanced melanoma patients, 200 PEMBRO and $200 \mathrm{IPI}+\mathrm{NIVO}$, were randomly selected from 12 US academic and satellite clinics that had both treatment options available.

- The study found that more frail patients (Eastern Cooperative Oncology Group score of 2-3) were more likely to receive PEMBRO, a treatment regimen with lower occurrence of toxicity in trials versus that of IPI+NIVO.

- Patients with BRAF wild-type or PD-L1 positive tumors are associated with PEMBRO monotherapy, irrespective of frailty.

- Patients prescribed PEMBRO or IPI+NIVO are otherwise similar with respect to demographics, income, education, insurance status, comorbidity index, metastasis, line of therapy, disease stage, presence of metastasis and lactate dehydrogenase elevation.

- As future real-word comparative effectiveness outcomes are examined, understanding these differences impacting treatment selection will be important.

Supplementary data

To view the supplementary data that accompany this paper please visit the journal website at: www.futuremedicine.come/doi/su ppl/10.2217/imt-2018-0150

Author contributions

RW Joseph: conceptualization, result interpretation, writing - review and editing; AC Shillington: methodology, investigation, formal analysis, visualization, project administration, writing - original draft; C Macahilig: methodology, investigation, resources, data curation, project administration, supervision, writing - review and editing; SJ Diede: conceptualization, result interpretation, writing - review and editing; $V$ Dave: resources, data curation, project administration, writing - review and editing; Q Harshaw: formal analysis, software, writing - reviewing and editing; FX Liu: conceptualization, supervision, result interpretation, funding, acquisition, writing - review and editing.

Financial \& competing interests disclosure

AC Shillington and Q Harshaw are employees of EPI-Q Inc. V Dave and C Macahilig are employees of Medical Data Analytics (MDA). EPI-Q and MDA received funding from Merck Sharp \& Dohme Corp., a subsidiary of Merck \& Co., Inc., Kenilworth, NJ, USA to conduct this study. EPI-Q and MDA received funding from Kite, Bristol-Myers Squibb (BMS) and Janssen Scientific Affairs outside this work. SJ Diede and FX Liu are employees of Merck Sharp \& Dohme Corp., a subsidiary of Merck \& Co., Inc., Kenilworth, NJ, USA. RW Joseph is a consultant to Merck \& Co., BMS (outside this work). The authors have no other relevant affiliations or financial involvement with any organization or entity with a financial interest in or financial conflict with the subject matter or materials discussed in the manuscript apart from those disclosed.

This manuscript was written by the authors. Authors received no payment for authorship. Medical Data Analytics and EPI-Q Inc. received funding from Merck \& Company for developing, formatting and submission of this manuscript.

Ethical disclosure

This study received exempt review and approval from the New England IRB.

\section{Open access}

This work is licensed under the Attribution-NonCommercial-NoDerivatives 4.0 Unported License. To view a copy of this license, visit http://creativecommons.org/licenses/by-nc-nd/4.0/ 


\section{References}

Papers of special note have been highlighted as: $\bullet$ of interest

1. American Cancer Society: Cancer facts and figures 2018 (2018). www.cancer.org/content/dam/cancer-org/research/cancer-facts-and-st atistics/annual-cancer-facts-and-figures/2018/cancer-facts-and-figures-2018.pdf

2. National Cancer Institute. Surveillance, Epidemiology, and End Results (SEER) cancer stat facts: melanoma of the skin (2018). https://seer.cancer.gov/statfacts/html/melan.html

3. Schachter J, Ribas A, Long GV et al. Pembrolizumab versus ipilimumab for advanced melanoma: final overall survival results of a multicentre, randomised, open-label Phase III study (KEYNOTE-006). Lancet 390(10105), 1853-1862 (1994).

- Pivotal trial on pembrolizumab (PEMBRO) as a single agent in this patient population.

4. Wolchock JD, Chiarion-Sileni V, Gonzalez R et al. Overall survival with combined nivolumab and ipilimumab in advanced melanoma. N. Engl. J. Med. 377(14), 1345-1356 (2017).

- Pivotal trail on PEMBRO versus ipilimumab plus nivolumab as a single agent in this patient population.

5. O. Hamid C, Robert A, Daud FS. 5-year survival outcomes in patients (pts) with advanced melanoma treated with pembrolizumab (pembro) in KEYNOTE-001. Presented at: 2018 ASCO Annual Meeting. Chicago, IL, USA, 28 November-2 December 1994.

6. Merck \& Company. Keytruda package insert. (2018) www.merck.com/product/usa/pi_circulars/k/keytruda/keytruda_pi.pdf

7. Bristol-Myers Squibb. Opdivo package insert. (2018) https://packageinserts.bms.com/pi/pi_opdivo.pdf

8. Kilbridge KL, Weeks JC, Sober AJ et al. Patient preferences for adjuvant interferon $\alpha-2 b$ treatment. J. Clin. Oncol. 19(3), 812-823 (2013).

9. Krammer R, Heinzerling L. Therapy preferences in melanoma treatment - willingness to pay and preference of quality versus length of life of patients, physicians and healthy controls. PLoS ONE 9, e111237 (2014).

10. Liu FX, Witt EA, Ebbinghaus S. Patient and oncologist preferences for attributes of treatments in advanced melanoma: a discrete choice experiment. Patient Prefer. Adherence 11(2017), 1389-1399 (2017).

- Patient reported study demonstrating preference for lower toxicity treatment as a trade off for survival in this patient population.

11. Heo M, Leon AC. Performance of a mixed effects logistic regression model for binary outcomes with unequal cluster size. J. Biopharm. Stat. 15(3), 513-526 (2015).

12. Robinson WS. Ecological correlations and the behavior of individuals. Am. Soc. Rev. 15(3), 351-357 (1950).

13. Cowey CL, Liu FX, Black-Shinn J et al. Pembrolizumab utilization and outcomes for advanced melanoma in US community oncology practices. J. Immunother. 41 (2), 86-95 (2018).

- First real-world study of PEMBRO in this patient population.

14. Chapman PB, Hauschild A, Robert C et al. Improved survival with vemurafenib in melanoma with BRAF V600E mutation. N. Engl. J. Med. 364(26), 2507-2516 (2011). 
